\title{
Quelques échos sur les travaux de l'Association Internationale d'Hydrologie Scientifique $\left({ }^{*}\right)$
}

\section{Remarks on the work of the International Association of Scientific Hydrology}

\author{
PAR L. SERRA \\ pRÉSIDENT DE LA COMMISSION DES EAUX DE SURFACE DE L'A.1.H.S.
}

\begin{abstract}
La contribution des quatre grandes Commissions constituant l'A.I.H.S. a été très importante. On s'est limité ici à un rapide exposé el quelques commentaires sur les travaux de la Commission des Eaux de surface dont le programme était dans ses grandes lignes :

- Etude des sécheresses et bas débits.

- Ecoulement provenant de la fonte du manteau neigeux;

- Variabilité temporelle et spatiale des précipitations.
\end{abstract}

\begin{abstract}
The contribution of the four big Commissions forming the Association was very substantial. This article is restricted to a brief summary of the Surface Water Commission's work, with a few additional remarks. The broad outlines of the Commission's programme were as follows: 1) Study of droughts and low-water flows;

2) Flow due to melting snow;

3) The variability of precipitations in time and space.
\end{abstract}

La présentation d'un ensemble de travaux constituant le thème et la matière d'une Assemblée générale ou d'un Congrès, est toujours une tâche ingrate et difficile.

C'est cependant devenu pour moi une sorte d'habitude de venir, après chacune des assemblées générales de l'Union Géodésique et Géophysique Internationale, vous apporter quelques échos sur les travaux de l'Association Internationale d'Hydrologie Scientifique qui est, comme vous le savez, une des sept associations qui com. posent l'Union.

En 1951, je vous avais fait part de ce qui s'était dit à l'assemblée de Bruxelles. En 1954, puis en 1957 , ce furent les comptes rendus des assemblées de Rome et de Toronto. Aujourd'hui, il s'agira d'Helsinki.

(*) Mémoire présenté à l'Union Géodésique et Géophysique Internationale, XII ${ }^{e}$ Assemblée générale, Helsinki, 25 juillet-6 août 1960 .
L'U.G.G.I., vous le noterez en passant, voyage pas mal. La prochaine assemblée générale, celle de 1963 , se tiendra en Californie.

Mais je reviens à notre assemblée d'Helsinki. Voici d'abord quelques chiffres qui vous montreront l'importance de cette manifestation.

- Plus de 1600 participants venus des quelque cinquante nations qui adhèrent à l'U.G.G.I : ceci pour le côté matériel;

- Sur le plan scientifique, et pour notre Association d'Hydrologie seule, près de 300 rapports ou communications remis au Secrétariat général pour présentation et discussion.

Et ceci appelle deux remarques préliminaires :

Les assemblées générales de l'U.G.G.I. sont tenues en principe par des géodésiens, des géophysiciens, disons d'une façon schématique par des «hommes de science » qui, par vocation, 
apportent tous leurs soins à la recherche fondamentale.

Mais on observe de plus en plus, et cela est particulièrement net en hydrologie, qu'un nombre croissant d'ingénieurs, soucieux de l'utilisalion la meilleure et la plus rationnelle de l'eau, s'intéressent et participent activement aux travaux de l'Association d'Hydrologie Scientifique. Tant il est difficile de dire, en ce domaine comme en bien d'autres d'ailleurs, où finit la recherche fondamentale et où commence la recherche appliquée.

C'est cet apport nouveau qui explique sans doute l'accroissement du nombre de communications que l'on constate à chacune de nos assemblées générales - et qui me conduit à la deuxième remarque :

En 1951, à Bruxelles, on avait enregistré environ 150 communications. Il y en a eu 300 à $\mathrm{Hel}$ sinki, d'où l'on peut conclure que le volume des travaux hydrologiques semble suivre à peu près la même loi que celle que l'on a pu observer pour la production de l'énergie électrique, c'est-à-dire la loi du doublement tous les dix ans.

S'il faut s'en réjouir, car il est ainsi prouvé que l'hydrologie est une science bien vivante, cela pose tout de même de sérieux problèmes en ce qui concerne du moins l'organisation des Assemblées Générales.

Comme on ne dispose en effet. lors de ces manifestations, que de sept à huit journées pouvant être consacrées à des séances de travail effectif, il est facile de voir qu'il faudrait, pour tenir les délais, pouvoir présenter et discuter une moyenne de six communications à l'heure, ce qui est une performance à peu près irréalisable si l'on tient compte surtout des différences de langage : à Helsinki on a parlé anglais - ou américain, ce qui est pire -- à peu près à $70 \%$.

La présentation, et plus encore la discussion des 300 rapports dont je vous ai parlé, en ont souffert quelque peu. Et si je voulais à mon tour faire un commentaire, si bref fût-il, sur chacun d'eux, je n'aurais pas trop de deux ou trois de nos séances. Je dois donc me limiter, et pour cela, laissant de côté tout ce qui a trait aux Eaux souterraines et aux Neiges et Glaces, je ne parlerai que des seuls travaux de la Commission des Eaux de surface.

Cela représcnte tout de même une centaine de rapports à examiner. Je dois dire tout de suite qu'ils ne sont pas tous d'égale valeur. Certains des monographies ou des études trop particulières - ne dépassent pas le niveau du « travail de bon élève» et l'on peut même s'étonner qu'ils aient eu les honneurs d'une présentation dans une assemblée internationale. Des mesures ont d'ailleurs été envisagées à Helsinki pour que cela ne se reproduise plus.

Je m'en tiendrai donc aux notes et commu- nications qui m'ont paru présenter le plus d'intérêt.

Le programme proposé par la Commission des Eaux de surface pour l'Assemblée d'Helsinki était basé sur trois thèmes principaux :

.... L'étude des bas débits et sécheresses (cette question a d'ailleurs fait l'objet d'un symposium tenu conjointement par notre Association, et l'Association Internationale de Météorologie) ;

-. L'étude de l'écoulement provenant de la couverture neigeuse;

- Et, en ce qui concerne les précipitations, l'étude de leur variabilité temporelle et spatiale.

Premier: sujet: La sécheresse.

Il s'agit d'un phénomène, moins spectaculaire que les crues et pour cette raison sans doute, un peu moins étudié, mais qui est tout de même très important et aussi très complexe.

Cette complexité, celle qui concerne aussi bien l'intensité des sécheresses que leur répartition et leur extension dans l'espace et dans le temps, est due à la fois à l'insuffisance des données d'ohservations et au fait que les causes physiques du phénomène sont encore imparfaitement connues.

Avec des données d'observation, on peut faire de la statistique. Si l'on s'intéresse aux facteurs qui occasionnent les sécheresses et aux lois reliant les causes à l'effet, l'étude devient plus «physique». Ce sont en somme ces deux grandes voies de recherche que l'on retrouve dans les communications présentées à Helsinki. Il convient de noter d'ailleurs, mais ceci n'est pas une nouveauté, qu'il est souvent difficile de faire le départ entre ce qui est purement statistique ct ce qui peut prendre un caractère plus physique.

Commencons donc par les statisticiens.

M. SNeyers, un Belge, a cherché à déterminer la probabilité d'un mois sans précipitation d'après les données recueillies à l'Observatoire d'Uccle. Des ajustements au moyen d'une loi de distribution $\Gamma$ incomplète ou d'une loi logarithmico-normale ne conduisent pas à des résultats satisfaisants. Par contre, la représentation de la distribution observée au moyen d'une branche d'hyperbole sur un graphique à échelle de probabilité normale lui apparaît comme excellente.

Le problème de la sécheresse est, comme vous le voyez, abordé ici sous un aspect particulier : M. Sneyers, en effet, se donne une durée fixe, le mois, et recherche la probabilité d'apparition d'un mois sans précipitation. 
Pour M. Szigyarto, de Budapest, qui présente les résultats d'une recherche des régularités earactérisant le développement de la durée annuelle des périodes sans pluie sur la grande plaine hongroise, c'est un aspect un peu différent.

La population des observations de la durée des périodes sans pluie commençant à une date « $t$ » arbitrairement choisie dans l'année, est considérée comme un ensemble d'échantillons qui, avec une approximation suffisante, sont indépendants et suivent une même loi de distribution. Or, cette loi serait exponentielle.

Ceci étant établi, c'est-à-dire les paramètres qui la caractérisent étant calculés, M. SzigYARTo examine la relation qu'il peut $\mathrm{y}$ avoir entre cette loi et l'instant $t_{0}$, c'est-à-dire l'époque de l'année choisie comme point de départ de la longueur des périodes sous pluie. Et il trouve une sorte de périodicité qui aurait pour amplitude environ un an et demi.

Ces calculs s'appliquent, je le rappelle, à ce qu'il considère comme une région caractéristique de la grande plaine hongroise. Il serait sans doute intéressant d'examiner si c'est là une particularité de cette région ou s'il s'agit d'une loi de portée plus générale.

Quoi qu'il en soit, cette note fait état de durées plus ou moins longues de sécheresse, ce qui, en fait, conduit à la notion de persistance La persistance est une des caractéristiques essentielles du phénomène au même titre que l'intensité, ce qui m'a du reste conduit à proposer, dans une note que j'ai moi-même présentée à Helsinki, de définir la sécheresse par un indice qui serait proportionnel au produit :

\section{intensité $X$ durée.}

Cette notion de persistance a été également soulignée par d'autres auteurs.

C'est ce que fait en particulier M. WEMELSFELDER, un Hollandais. Il remarque, d'après un certain nombre d'exemples, que si les précipitations mensuelles sont des variables alćatoires, c'està-dire se distribuant suivant les lois du hasard, il se superpose pour les débits (considérés eux aussi à l'échelle mensuelle) une autre loi, celle de la persistance.

En désignant simplement par + et par - les mois au cours desquels les débits sont supérieurs ou inférieurs à la normale, on trouve pour les séquences de mois de même signe (donc pour les sécheresses qui se prolongent lorsqu'il s'agit du signe -) des durées plus longues si l'on considère les débits, que si l'on considère les précipitations.

Autrement dit, lorsqu'une sécheresse « en débit », c'est-à-dire une hydraulicité faible, dure depuis quelque temps, la probabilité pour qu'elle continue va en augmentant.
Les dates de début des persistances + ou restent cependant soumises aux lois du hasard.

Pour Kritzky et Mrrkel de l'U.R.S.S., il existe une certaine corrélation entre les débits annuels eux-mêmes. Les fluctuations annuelles du débit suivent une loi de fréquence que l'on peut, après quelques décennies d'observation, estimer plus ou moins exactement par le calcul de la moyenne et de l'écart-type. Mais il faut $y$ ajouter un coefficient de dissymétrie. Pour un très grand nombre de rivières, la loi de distribution s'ajusterait asssez bien suivant une loi du type III de Pearson, avec un coefficient de dissymétrie égal à deux fois le coelficient de variation.

Cela nous rappelle, entre parenthèses, les travaux de M. HALPHEN qui avait proposé pour les rivières françaises des lois de distribution dont la forme analytique était une génćralisation des lois de Pearson.

Pour en revenir à Kritzky et Merkel, ils montrent qu'entre les débits annuels successifs, il existe une corrélation non négligeable. La séquence des débits annuels forme une chaîne de Markow, et le coefficient de corrélation peut atteindre 0,4 et parfois même 0,5 . D'où la tendance à observer des séquences d'années de même nature - humides ou sèches - ce qui rejoint les conclusions de M. Wemelsfelder.

Une dernière remarque maintenant avant d'abandonner cet aspect purement statistique de la question.

L'ćtude exhaustive des sécheresses exige, on s'en doute bien, une énorme documentation, souvent difficile à constituer si l'on désire des séries d'observation suffisamment longues.

Mais que doit-on entendre par séries «suffisamment longues »? En d'autres termes, quelle doit être la longueur minimale d'une série d'observations?

Il arrive aussi parfois, mais le cas est plutôt. rare, que l'on dispose précisément d'une longue série d'observations. Pour Paris, par exemple, on connait les précipitations mensuelles depuis 1688 , c'est-à-dire, compte tenu de quelques lacunes, depuis deux siècles et demi au moins, ce qui fait une série de quelque 3000 valeurs mensuelles: le rêve de tout statisticien.

Mais là se pose une autre question : n'y a-t-il pas lieu de tenir compte d'une possible évolution de la pluviosité à longue période? et plutôt que de parler de la longaeur minimale d'une série d'observations, ne conviendrait-il pas de faire appel plutôt à une autre notion : celle de la lonsueur optimale d'une série?

C'est un point que j'ai signalé à Helsinki; je ne puis, faute de temps, m'y étendre plus longuement aujourd'hui, mais cela pourra faire, à l'une de nos prochaines réunions du Comité technique, l'objet d'une autre communication.

J'en viens maintenant à une deuxième séric 
d'études : celles à caractère plus physique que mathématique.

Je citerai d'ahord - mais sans pouvoir non plus m'y attarder bien longtemps - M. ZsuFfa qui décrit comment se font en Hongrie les prévisions à longue échéance des débits d'étiage en saison sìche. Une partie de ces débits est due aux eaux qui se sont accumulées dans le sous-sol et qui proviennent des précipitations tombées en hiver. Zsuffa détermine les coefficients de Fourier des hydrogrammes provenant de l'eau emmagasinée, ce qui lui permet d'avoir l'enveloppe inférieure de l'hydrogramme d'été, d'où la possibilité, moyennant certaines conditions à caractère local, de prévoir les bas débits à échéance d'un mois. Les résultats sont, parait-il, très satisfaisants.

Etude du même genre de Mann et Rasmusson du Weather Bureau des Etats-Unis qui indiquent comment se font certaines prévisions de bas débits sur le Mississippi. Il s'agit là d'un problème d'utilisation du cours inférieur de ce fleuve pour la navigation et ce qu'il importe de savoir, ce sont les périodes où le trafic par chalands et remorqueurs est possible. Les moyens mis en œuvre sont à l'échelle de l'Amérique : ce sont les données de 533 pluviomètres enregistreurs, les hydrogrammes unitaires de 208 bassins versants élémentaires, plus des prévisions de précipitations et de températures, la connaissance de tous les programmes d'utilisation des réservoirs à l'amont, etc., le tout enfourné dans des machines électroniques qui permettent d'émettre tous les mercredis un bulletin de prévision des bas débits couvrant une période d'un mois.

Actuellement, les prévisions diffèrent de la réalité de 25 à $30 \%$ en moyenne, mais des améliorations sont escomptées.

Laszloffy, en Hongrie, utilise plus simplement des diagrammes établis d'après les valeurs observées et qui donnent la relation entre le débit moyen et le débit des basses eaux sur une longue période. Ces relations varient avec la nature des terrains considérés, ce qui le conduit à l'établissement d'abaques. Il semble qu'il y ait là une idée intéressante et sans doute cela vaudrait-il la peine que nous tentions de faire un travail analogue pour nos cours d'eau français, complété, comme le fait Laszloffy, par une carte donnant les débits d'étiage moyens spécifiques pour des bassins de 100 à plusieurs milliers de kilomètres carrés.

Et cette carte me fournit une transition toute naturelle pour vous dire maintenant quelques mots sur les études relatives à la répartition spatiale des sécheresses.

M. RodIER, prenant l'exemple de la sécheresse exceptionnelle observée dans les régions équatoriales en 1958, a montré comment elle s'était étendue suivant une bande parallèle à l'équa- teur, exactement d'ailleurs comme les périodes sèches observées en 1913 et en 1941-1945.

Mais je n'insisterai pas là-dessus, M. RonIer pourra sans doute vous en parler plus abondamment, et mieux que je ne saurais le faire, au cours d'une séance prochaine.

Un autre exemple d'extension des sécheresses dans l'Illinois a été présenté par HuFF et CHAIGNON, et sur les grandes prairies canadiennes par LAYCOCK.

J'ai essayé pour ma part d'indiquer, par une représentation à deux dimensions, comment avait varié dans l'espace et aussi dans le temps, l'hydraulicité observée au cours de trente années sur huit bassins caractéristiques de l'Europe répartis du Portugal à la Russie.

\section{Il est apparu de cette représentation :}

- D'une part, qu'il ne semblait pas y avoir entre bassins pris deux à deux, des fluctuations parallèles de l'hydraulicité, donc en particulier aucun synchronisme apparent des périodes sèches;

- Et que, d'autre part, il ne semblait se dégager aucune loi en ce qui concerne la répartition dans le temps des années sèches et humides - d'où la conclusion qu'il faut à mon sens renoncer à vouloir trouver à tout prix dans le régime des cours d'eau des cycles ou périodicités.

Il s'agit là - je parle des périodicités - d'une question qui demeure très controversée. Voici très brièvement ce que, pour ma part, j'ai dit à Helsinki :

Aucun cycle réel dans les fluctuations de l'hydraulicité ou de la pluviosité n'a jusqu'à présent pu ètre scientiliquement prouvé. C'est un fait. Mais si la périodicité du phénomène global considéré en tant que tel n'existe pas, il n'est pas interdit de penser qu'une périodicité peut exister dans les causes qui le provoquent.

Il faut donc remonter aux causes météorologiques de la pluie, à ces caractères physiques dont on se rend compte, chaque jour davantage, qu'ils doivent servir de support à toute étude vraiment complète du phénomène. Ces causes sont ici les variations du champ de pression qui détermine l'apparition et l'évolution des anticyclones et des zones dépressionnaires, lesquels sont associés aux temps secs et aux temps à pluie, et dans ces causes il y a effectivement un rythme.

Tout cela, bien entendu, mériterait de plus amples développements. Mais mon rôle aujourd'hui n'est pas d'expliquer ou de défendre des théories, mais simplement de mentionner - trop rapidement d'ailleurs - les travaux présentés à Helsinki.

Je poursuis donc mon exposé en vous signalant maintenant une étude très intéressante d'un mé- 
téorologiste américain, NAMIAS, sur les facteurs d'initiation, d'évolution et de fin des sécheresses.

La sécheresse dans les latitudes tempérées, surtout celle d'été, est généralement liée à des anticyclones mi-troposphériques donnant des pressions supérieures à la normale. Les anticyclones sont asymétriques au point de vue de la répartition en humidité : ils seraient, d'après Namias, composés de langues humides et sèches s'enroulant intérieurement en spirales, les langues seches ayant leur origine dans les niveaux supérieurs des vents d'ouest déplacés vers le nord et descendant dans le noyau à haute pression. Semblables dislocations ou anomalies dans les vents d'ouest se produisent presque toujours en groupes plutôt qu'isolément, de telle sorte qu'un certain nombre de zones de sécheresse interdépendantes se développent dans le même temps.

Je crains qu'un résumé aussi bref des travaux de Namias ne permette pas d'en saisir tout l'intérêt. Je crois pour ma part qu'il y a là l'explication de cette répartition en bandes plus ou moins allongées de la sécheresse que j'ai pu constater sur divers pays de l'Europe, que M. Rodier a signalée pour l'Afrique Equatoriale et qui est sans doute un phénomène très général.

\section{Que pourrai-je vous signaler encore?}

D'abord un travail de Baranof et Popor sur le calcul des débits minimaux des cours d'eau inexplorés.

Il existe en effet sur le territoire de l'U.R.S.S. un certain nombre de cours d'eau où aucune mesure des débits n'a encore été faite, mais qu'il serait utile de connaitre en vue de leur aménagement. La méthode consiste à établir des formules empiriques correlant les minimums mensuels et journaliers avec les débits annuels moyens et d'autres données climatologiques, ceci pour tous les bassins où des mesures ont été faites, et d'appliquer ces formules à ceux où il n'existe que peu ou pas de mesures. Il faut, bien entendu, que ces groupes de bassins soient dans des conditions générales « homogènes ».

Il paraîtrait que les résultats ainsi obtenus sont assez satisfaisants. Bien que restant très approximatifs, ils valent tout de même mieux que pas de résultats du tout.

Dans un ordre d'idées assez voisin, nous avons eu aussi une note de LANGBEIN, du Weather Bureau des Etats-Unis, et qui a, semble-t-il, à peu près les mêmes préoccupations que les Russes.

Dans les régions aux territoires en voie d'équi. pement, mais où les données d'observation et les études hydrologiques sont encore insuffisantes, se pose souvent le problème de la validité des moyennes de précipitations et d'écoulement obtenues au moyen de périodes d'observation courtes.
L'idée de LANGBein est que certaines données sur le niveau des eaux souterraines ou même le niveau des lacs peuvent servir d'index pour déterminer la précipitation ou le ruissellement correspondant à de longues périodes, c'est-à-dire ce que l'on appelle communément les valeurs moyennes.

La nappe d'eau souterraine ou le lac de surface sont des réservoirs ayant un temps de réponse long et qui intègrent les effets de la précipitation sur des intervalles de temps très prolongés. Le temps de réponse est défini par le rapport du changement de volume d'eau emmagasiné au changement correspondant du débit sortant. La variation du débit sortant du réservoir est inversement proportionnelle au temps de réponse.

Dans les exemples cités par LangBein, le facteur d'amplification des périodes d'observation varie de trois à quatre. Autrement dit, il faut, pour déterminer les valeurs moyennes de la précipitation ou de l'écoulement, quatre fois moins de temps que par les méthodes classiques, el cela avec une précision comparable.

Quant à moi, c'est quatre fois plus de temps qu'il m'aurait fallu pour vous dire tout ce qui s'est passé à Helsinki. Car après les sécheresses on a parlé aussi - et il ne s'agit toujours que de la seule Commission des Eaux de surface - de l'écoulement provenant de la couverture neigeuse, et des précipitations.

Sur l'écoulement dû à la couverture neigeuse, peu de nouveautés à signaler, à part peut-être une note de GLason et Anderson indiquant comment peut varier le débit de fonte sur un bassin versant boisé suivant la façon d'exploiter la forêt.

Is ont étudié dans la Sierra-Nevada de Californie l'influence de divers types de coupes : par exemple, forêt rasée sur une largeur de l'ordre de deux hauteurs d'arbres adjacents, ou coupe en bloc des gros arbres, ou enfin coupe sćlective des arbres de diamètre supérieur à $1 / 2$ mètre, les autres étant laissés en place. L'expérimentation a porté sur deux années, ce qui est peut-être un peu court. Je ne vous donnerai pas le détail des résultats, mais seulement leur ordre de grandeur qui est à peu près le suivant : l'augmentation d'eau accumulée sous forme de neige aurait été de l'ordre de 150 à $200 \mathrm{~mm}$ par an si on compare ces nhservations à celles faites sur la forêt adjacente et non attaquée. Autre résultat, mais encore provisoire lui aussi : l'enlèvement des broussailles aurait diminué les pertes estivales d'eau d'une quantité de l'ordre de 100 à $150 \mathrm{~mm}$.

On peut dire en définitive que la production d'eau en provenance d'une zone enneigée peut être influencée à la fois en quantité et dans sa répartition dans le temps par la manière dont on 
exploite la forêt et même les broussailles. Cela doit intéresser les forestiers et les hydrologues.

$$
* * *
$$

Le dernier chapitre de mon exposé concernera les travaux du Comité des Précipitations.

Le programme mis à l'étude pour l'assemblée d'Helsinki comportait essentiellement trois points :

- les observations et mesures;

- la variabilité des précipitations dans le temps et dans l'espace;

- et enfin les méthodes de mesure spéciales telles que le radar.

En fait, ces trois points étaient articulés autour d'une idée centrale : l'étude de la variabilité.

Plutôt que de vous donner le détail de la douzaine de communications présentées - et qui seront, comme toutes les autres d'ailleurs, publiées in extenso — je vais plutôt essayer de vous faire part des idées générales qui s'en dégagent.

La précipitation est une quantité d'eau qui tombe sur le sol, c'est-à-dire sur une surface. Ce que l'on observe, c'est la quantité d'eau qui tombe sur un point: le pluviomètre. Ce n'est donc qu'un échantillon.

Or, cet échantillon dépend non seulement de la nature même de la pluie (variable comme on le sait d'un lieu à l'autre, même s'ils sont voisins), mais encore de la façon dont on fait la mesure, c'est-à-dire de la forme même du pluviomètre çui dévie plus ou moins les filets d'air et les gouttes de pluie, de sa hauteur, de son environnement, etc.

On est donc conduit tout naturellement à penser que la «précision» dans la connaissance de la quantité d'eau effectivement tombce sur une région s'accroìtra davantage par la multiplication des échantillons, c'est-à-dire par l'extension des réseaux pluviométriques, que par les perfectionnements apportés aux appareils eux-mêmes.

Autrement dit, il est illusoire de rechercher la précision du dixième de millimètre sur un point de mesure, si l'on est obligé de faire sur les points avoisinants des approximations qui sont souvent assez grossières.
D’où l'idée exprimée par le président du Comité des Précipitations et développée par le météorologiste suédois Thor Bergeron (en exposant les résultats de ses travaux) qu'il conviendrait pour chaque étude de doubler les réseaux climatologiques existants par des réseaux «temporaires» spécialisés, d'installation et d'exploitation peu coûteuses, et qui, permettant l'analyse détaillée des situations météorologiques typiques et des phénomènes qui lui sont associés, nous ferait avancer plus rapidement et plus sûrement dans la voie de la connaissance.

Une autre idée, qui s'était déjà dégagée des séances de travail sur les sécheresses et qui rejoint d'ailleurs celle que je viens d'évoquer à propos des précipitations, c'est la nécessité de s'orienter plus qu'on ne l'a fait jusqu'à présent vers l'étude physique de ces manifestations naturelles.

On peut dire en effet qu'à l'origine, lorsqu'on ne connaît pas encore grand-chose d'un phénomène, on procède à des mesures, et à travers les cas particuliers que l'on observe chaque jour, on cherche à dégager des tendances générales. C'est le stade des « moyennes », c'est-à-dire de la Statistique plus ou moins élaborée.

Mais ensuite, et lorsqu'on en a acquis une vue d'ensemble, il devient intéressant d'analyser avec plus de détails chaque cas particulier et de rechercher pour quelles raisons et sous l'influence de quels facteurs ils s'écartent du cas général. C'est la seule façon de comprendre et de savoir.

$$
* *
$$

J'aurais eu encore beaucoup à dire sur ce sujet et d'une façon plus générale sur la somme des travaux qui ont été présentés à Helsinki. J'espère que ce compte rendu trop bref à mon gré, et par la force des choses un peu décousu, vous aura tout de même montré combien cette manifestation de l'Association Internationale d'Hydrologie Scientifique a été intéressante.

Grâce à l'activité inlassable de notre secrétaire général M. Tison, que beaucoup d'entre vous connaissent bien, toutes les notes ou communications présentées sont ou seront publiées intégralement.

Nous aurons donc tout loisir pour les relire et les méditer. Ett c'est là que commencera véritablement le travail intéressant. 


\section{I S C US S I O N}

président : M. ReMENieras

M. le Président remercie M. Serra de son exposé et espère qu'en raison des nombreux sujets intéressants abordés, celui-ci suscitera diverses questions.

M. DE Rouville indique que le conférencier se plaint de la nature ponctuelle des pluviomètres comme faussant probablement les mesures de la quantité moyenne de pluie tombée dans la région environnante.

$\mathrm{Ne}$ pourrait-on pas tirer des impluvia des villages en zone sèche (Afrique du Nord, par exemple) une mesure moyenne plus satisfaisante de l'eau tombée sur une surface plus vaste? Les impluvia des phares isolés sont lissés, pentés et bétonnés et ne laissent qu'une faible prise à l'action de l'absorption par le sol et l'évaporation.

M. Serra dit que, le plus souvent, le bassin versant, lorsqu'il est équipé d'une station de jaugeage, constitue un pluviomètre intégrateur. Contrairement à ce qui est possible pour les impluvia des phares, ce pluviomètre ne mesure pas exactement la quantité de pluie qui tombe, car l'écoulement en sol naturel est conditionné non seulement par la pluie mais aussi par tout ce qui passe à l'intéricur du sol et tout ce qui s'évapore et ne peut être négligé.

M. le Président pense que si, dans certains cas, le pluviomètre ne mesure pas exactement la hauteur d'eau tombée, son indication est un index qui est en corrélation plus ou moins étroite avec ladite hauteur. Si l'on veut avoir un index valable, il faut que l'appareil de mesure soit toujours le même; d'où la nécessité de pluviomètres normalisés au moins pour les pluies fines et la neige.

M. Rodien fait trois remarques :

$1^{\circ}$ En Afrique comme en U.R.S.S, et aux U.S.A., on a été dans l'obligation de donner des valeurs-types pour les débits d'étiage des zones mal connues tels que : débits d'étiage pour cuirasses latéritiques, pour grès ordoviciens, etc. Mais ces chiffres ne sont valables que pour des bassins de superficie asse: grande, par exemple plus de $3000 \mathrm{~km}^{2}$. Pour des bassins plus petits, une seule résurgence suffit à fausser les faibles débits, même pour un terrain où les résurgences sont absolument normales.

$2^{\circ}$ Pour passer de la précipitation ponctuelle à la hauteur de précipitation sur une surface de dimensions moyennes, on a envisagé de placer des pluviomètres «Association» relevés à intervalles réguliers et de faible durée. Mais on peut également placer des pluviomètres totalisateurs de type simple qui permettent de tracer les isohyètes annuelles et qui ne nécessitent pas d'appointer les observateurs.

$3^{\circ}$ A propos de la variation de la hauteur de précipitation observée en fonction de la superficie de l'anneau du pluviomètre, il signale qu'en plusieurs endroits il a placé côte à côte un pluviomètre «Association» et un bac Colorado de $1 \mathrm{~m}^{2}$. Pour des averses observées très sérieusement, le bac Colorado donnait, par rapport au pluviomètre, un écart très faible, quoique systématique, de l'ordre de 4 à $5 \%$.

M. le Président remercie M. Ronen et rappelle qu'au Congrès d'Helsinki de l'A.I.H.S. on a souligné la tendance actuelle vers une collaboration plus intime entre Jhydrologie et la météorologic. On semble prendre davantage conscience du fait que les débits provienment de la pluie, que la pluie est liée aux perturbations atmosphériques et plus précisément aux «flux d'air humide». On assiste également à un renouveau d'intérêt pour les prévisions des débits moyens et extrêmes (M. Serra nous a parlé d'un mémoire hongrois qui traite de la prévision des étiages à partir de la courbe de tarissement).

\section{Goblot présente les remarques ci-après :}

«Les observations personnelles de M. Serna lui ont montré qu'il n'existait pas de cycle de pluviométrie et d'écoulement. D'autre part, M. SErna a signalé une communication sur ce qu'on peut tirer de l'étude des variations de niveaux des lacs ou des nappes souterraines profondes qui intègrent les effets des précipitations sur leurs bassins d'alimentation.

«Les niveaux des nappes peuvent être affectés par leur exploitation; celui des lacs européens, relativement petits, par les variations de l'évaporation qui peuvent être grandes dans nos climats «variables 》 et qui peuvent soit s'ajouter, soit s'opposer aux effets de variations de l'alimentation.

«La Caspienne, le plus grand des lacs avec ses $420000 \mathrm{~km}^{2}$, lac parfait, sans écoulement, est située dans une zone où la régularité des conditions d'èvaporation en été est remarquable et il semble qu'elle doit pouvoir bien intégrer les écoulements des différents bassins qui l'alimentent, dont le plus considérable est, de loin, la Volga.

«Se superposant à des variations saisonnières d'une amplitude de $0,80 \mathrm{~m}$ (maximum en juin, minimum en janvier), les observations faites au marégraphe de Bakou pendant un siècle, de 1831 à 1931 , montrent en janvier des cycles environ de 10 à 11 ans, dont les écarts sont inférieurs à ceux du cycle annuel :

Baisse de $0,70 \mathrm{~m}$ de 1837 à 1843 ,

- de $0,60 \mathrm{~m}$ de 1869 à 1873 ,

- de $0,70 \mathrm{~m}$ de 1917 à 1925 .

L'écart le plus grand pendant ce siècle était de $1,15 \mathrm{~m}$ entre un maximum de 1869 et un minimum de 1925 (3 cycles $1 / 2$ ).

"Il est vrai que depuis 1935 une baisse très forte, ayant dépassé $0,25 \mathrm{~m}$ certaines années, a été provoquée par les constructions du canal Don-Volga et les importants travaux faits par les Russes. Cependant aux travaux et aux causes purement climatiques peuvent s'ajouter d'importantes modifications des fonds du bassin sud situé dans une des zones les plus secouées du globe. Reste cependant la période séculaire de 9 ou 10 eycles assez réguliers, qui ne peut pas être écartée sans examen. 》

M. Gonlot demande à M. Snna si le fait qu'il n'ait pu observer de cycle ne vient pas de ce que les obserrations portent sur des bassins trop petits ef sur des périodes trop courtes.

M. SenRa répond que la taille du bassin ne lui semble pas être un facteur à prendre en considération pour ètudier l'existence de périodicités, car on peut aussi bien concevoir cette étude sur une aire très limitée cll se servant seulement d'observations «ponctuelles》.

La variable importante est ici le temps, c'est-à-dire la durée des observations. Mais, même avec de longues séries, on n'a pu parvenir à prouver la réalité des cycles. C'est ce que confirme précisément l'exemple cité par M. Goblot : pendant prés de cent ans, on a observé, ou cru observer, sur la mer Caspienne, un evele de dix à onze ans. Depuis 1935, on ne l'observe plus. L'intervention humaine (canal Volga-Don) n'étant pas suffisante pour expliquer un tel effet, il faut cn conclure qu'il s'agissait, en fait, d'une «apparence de périodicité » mais rien de physiquement réel.

M. Romen indique que, pour le $\mathrm{Nil}$, il $\mathrm{y}$ a eu un cycle pendant un certain temps, mais que ce eycle a ensuite disparu sans, pour cela, que l'on puisse, comme pour la Caspienne, l'imputer à des travaux humains. 
M. FERRY fait observer que l'analyse harmonique d'un phénomène variable quelconque sur une durée limitée permet toujours de le décomposer en une somme d'éléments périodiques. Par le seul fait du hasard, certaines de ces composantes peuvent paraître d'amplitude relativement importante. Mais en l'absence d'explication causale par des phénomènes physiques effectivement périodiques, rien ne prouve que les mèmes composantes se retrouveront dans une série temporelle ultérieure et sont utilisables pour des prévisions. Pour asseoir des prévisions sur une base purement empirique, il conviendrait de mettre en évidence les composantes périodiques éventuelles sur une durée au plus égale à la moitié de celles des observations connues et de vérifier leur persistance sur l'autre moitié des observations. Or, non seulement on utilise généralement la totalité des observations pour la recherche des composantes périodiques, mais on conslate que les périodes prédominantes trouvées sont souvent (en dehors des phénomènes diurnes ou saisonniers évidents) de l'ordre de $1 / 3$ ou de $1 / 4$ de la durée totale des observations, de telle sorte qu'une telle vérification serait peu significative.

M. Ie Président s'associe à ce que vient de dire MI. FERRY : il est toujours possible de décomposer n'importe quelle courbe observée en une série de Fourier; le problème est de savoir si, en extrapolant dans le temps la série de Fourier ainsi déterminée, on arrive à prévoir les phénomènes à venir avec une précision suffisante pour que la prévision ait un intérêt pratique.

M. Bonnin demande si le caractère fugitif des cycles observés ou contestés et leur période - 10 à 12 ans n'ont pas suggéré de chercher un rapprochement avec celui des taches solaires.

M. le Président dit que de nombreuses recherches ont été faites dans ce sens, mais sans grand succès, semble$\mathrm{t}$-il.

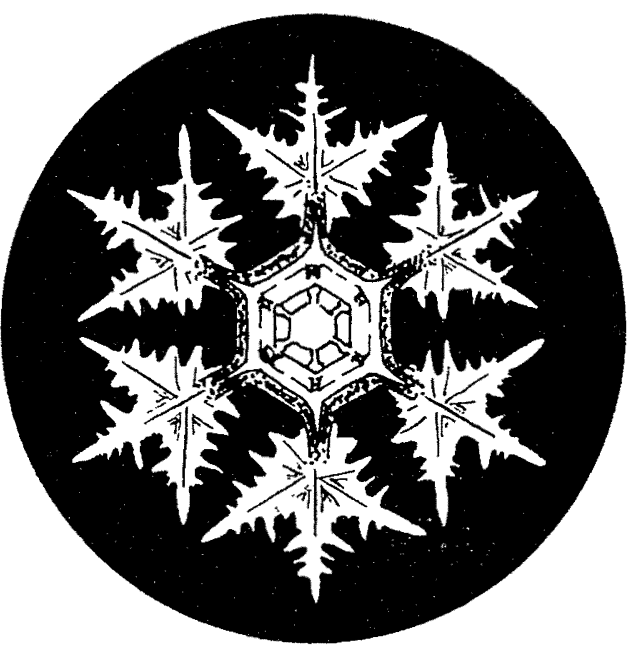

